\title{
Wireless Acoustic Emission Transmission System Designed for Fault Detection of Rotating Machine
}

\author{
Linghao Zhou ${ }^{\mathrm{a}}$, Fang Duan ${ }^{\mathrm{a} *}$, David $\mathrm{Mba}^{\mathrm{a}}$ \\ ${ }^{a}$ School of Engineering. London South Bank University, 103 Borough Road, SE1 OAA, London, UK
}

\begin{abstract}
Acoustic Emission (AE) has been adopted widely in structural health monitoring of buildings, walls, bridges, and has shown great potentials in incipient rotating machine fault detection [1, 2]. However, one inevitable problem that hinders $\mathrm{AE}$ of being practically applied in rotating machine fault detection is the transmission of AE signal inside the machine, as AE signal generally comprises high frequency components of typically $100 \mathrm{kHz}$ to $1 \mathrm{MHz}$ which will require transmission system to have extremely high data-rate for real-time monitoring. Traditional digital wireless system, for instance, Wi-Fi or Bluetooth, cannot satisfy the requirement. This paper addresses the issue of wireless AE transmission for rotating machine, with a modified analogue AE wireless transmission system. Piezoelectric wafer active sensor (PWAS), a labcrafted miniature sensor was adopted to fulfill the need of small system.
\end{abstract}

(C) 2012 Published by Elsevier Ltd. Selection and/or peer-review under responsibility of Global Science and Technology Forum Pte Ltd

Keywords: Acoustic Emission, Analogue Wireless Transmission, PWAS Sensor

\section{Introduction}

Acoustic Emission is generally described as transient elastic wave that generated from deformation or damage on the surface or within the material. It has been adopted in structural health monitoring due to the capabilities of monitoring internal incipient cracks of walls. In terms of rotating machine, AE sources are coming from gear meshing, bearing rolling, shaft rotating and all the other contacts between components which have relative motions. Defects such as gear tooth cracks, bearing race pitting can also significantly alter the waveform of AE signals so that incipient fault detection is theoretically feasible. Research conducted by Abdullah M. Al-Ghamd [3] and David Mba [2] has shown that AE can be adopted as effective method for

\footnotetext{
* Corresponding author. Tel.: +44 7448001133

E-mail address: duanf@1sbu.ac.uk.
} 
bearing fault detection, with unique advantage of estimating defect size and monitoring fault progression. Mathew et. al. [4] has applied AE inside helicopter main gearbox as mean of detecting bearing faults, and demonstrated promising results.

However, problems exist that prevent $\mathrm{AE}$ from being recognised as mainstream method for rotating machine health monitoring and fault detection. For starters, in order to get the best of AE's capability to react to incipient defect, AE sensor should be attached as close as possible near monitored rotating components, such that the AE signals generated from contacts of defects against rolling or meshing surfaces will not be attenuated too much by complex transmission path. Wireless transmission is hence mandatory, which is challenged by the inherent feature of $\mathrm{AE}$ signal being valid only in high frequency range. Typically, $\mathrm{AE}$ sensor reacts to excitations and generates signals of $100 \mathrm{kHz}$ to $1 \mathrm{MHz}$. Traditional digital wireless system can hardly handle real-time transmission of such high frequency contents. Even for high speed protocol such as Wi-Fi, the task can be cumbersome, considering the fact that $\mathrm{AE}$ signal sampled at $5 \mathrm{MHz}$ combined with 12 bit ADC could possibly require a system of handling 60Mbps for real time transmission. Grosse and Glaser $[5,6]$ developed wireless sensor network for AE system, Mistras Company offers commercial AE wireless monitoring system, but they all targeted off-line transmission and lower frequency range of AE signal. Space consuming of such system and AE sensors could also be an obstacle, as large system and sensor potentially endanger rotating machine if installed at rotating components.

Zahedi and Huang [7, 8] designed an interrogation-based analogue AE wireless system with commercially available components, which can transfer $\mathrm{AE}$ analogue waveform directly to overcome the shortcomings mentioned above. A lab-crafted AE sensor, namely PWAS is adopted for its miniature feature and reliable response. The system diagram is shown in Fig 1, it is a combination of two parts: Signal Interrogation Unit (SIU) and Sensor part. A high frequency $2.4 \mathrm{GHz}$ signal is separated to two paths, one is sent as Interrogation signal through SIU transmitter antenna after amplifying for signal modulation, the other one is sent to downconverting mixer for signal demodulation. In the sensor part, AE signal will be firstly buffered by voltage follower to compensate for high impedance of PWAS sensor, then mixed with the interrogation signal from SIU Transmitter antenna, and finally sent through the patch antenna at output port of the mixer. SIU receiver antenna will be tuned to receive the mixed signal, after band-pass and low-noise amplification, this signal will be demodulated using the same interrogation signal to recover the AE Signal. The process of digitalisation and cable transfer will then be undertaken.

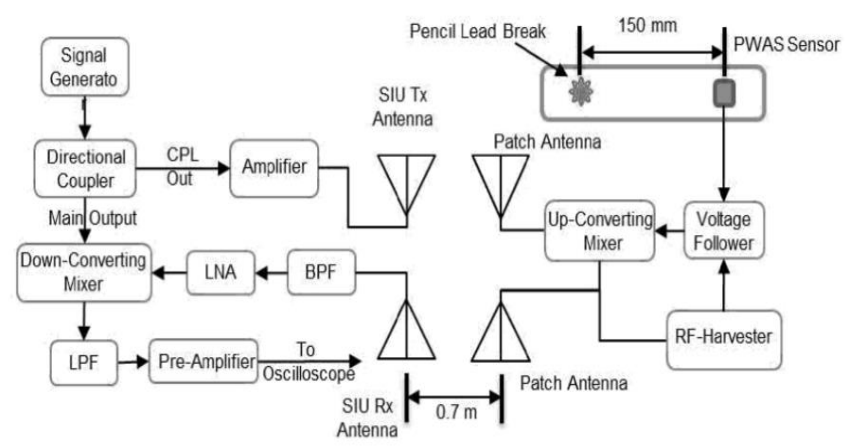

Fig 1 Analogue AE wireless transmission system [8] 


\section{Test of Analogue AE Transmission System}

Inspired by Zahedi's design, such system was set up first, several tests were then carried out to firstly validate the system's ability of wireless signal transmission, followed by testing the system's performance regarding antenna angle changes to simulate rotating condition. Pencil lead breaks were adopted as excitations of AE source. A commercial WD AE sensor was adopted as comparison and connected directly to 2/4/6 preamplifier and data card using cable. Both WD sensor and PWAS sensor were attached to a metal surface, and a pencil lead break (PLB) was performed at their middle point to excite a AE signal. Both signals were sampled at $4 \mathrm{MHz}$, pre-amplified by $40 \mathrm{~dB}$ and band passed between $100 \mathrm{kHz}$ and1200kHz. Signal trigger level was set to 45 to filter ambient noises.

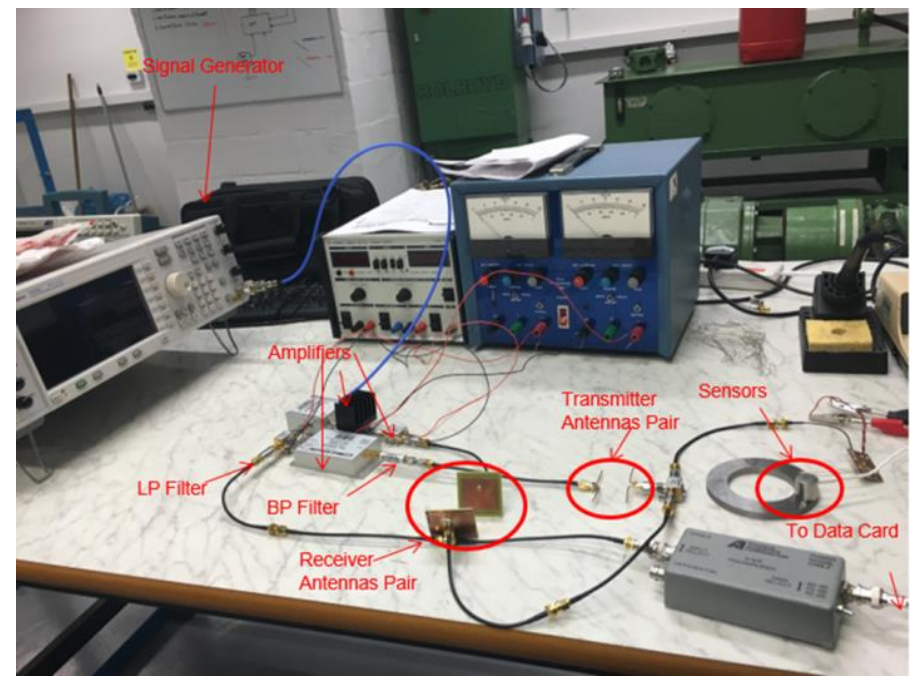

Fig 2 Analogue AE wireless transmission system set up
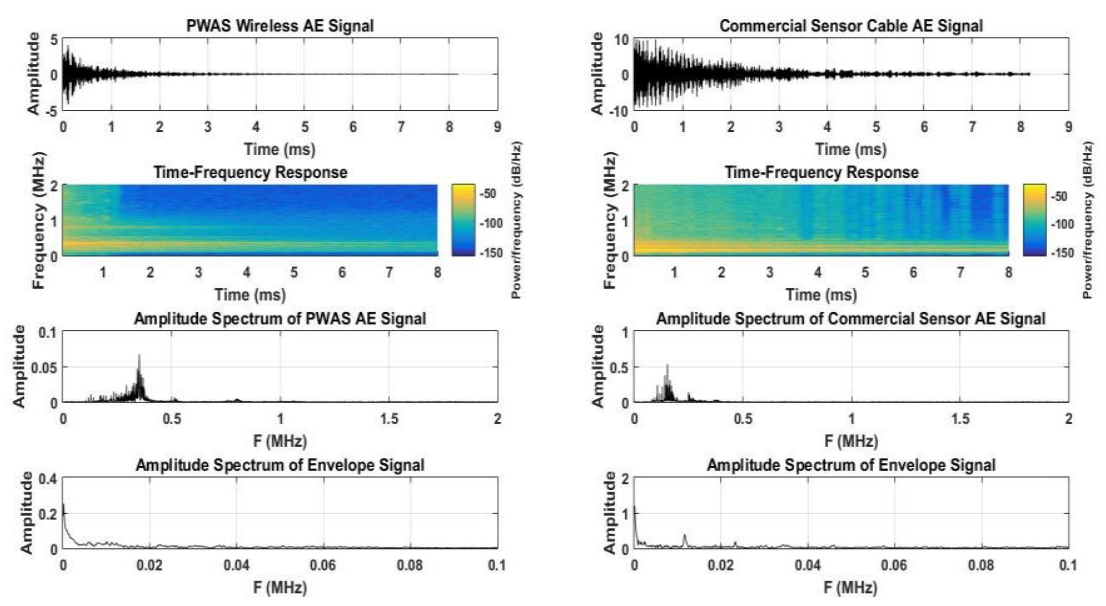

Fig 3 Comparisons of pencil lead break test of wireless signal and cabled signal 
The result shown in Fig 3 suggests that, wireless AE signal has similar frequency content and timefrequency response. The fact that wireless system can receive reactions from excitation means AE signal can be transmitted wirelessly through the designed system. The differences of response amplitude and envelope are due to amplifications and sensor types. In addition, later angle test with one pair of antennas fixed showed that the response of wireless signal remained flat in approximately $30^{\circ}$ angle range. With high sampling frequency of $5 \mathrm{MHz}$, considering rotating machine speed of hundreds rpm, enough points will be collected within the time of rotating component spinning for $30^{\circ}$.

\section{Test of Modified System for Rotating Machine}

However, such system was developed for long range transmission, thus miniature design was excluded when chose system components and antennas. In addition, this system contains two pair of antennas, which adds more uncertainties when the transmitter part is rotating with the machine. To address these issues, a modified miniature system with only one pair of antenna is proposed. The schematic diagram is depicted in Fig 4.

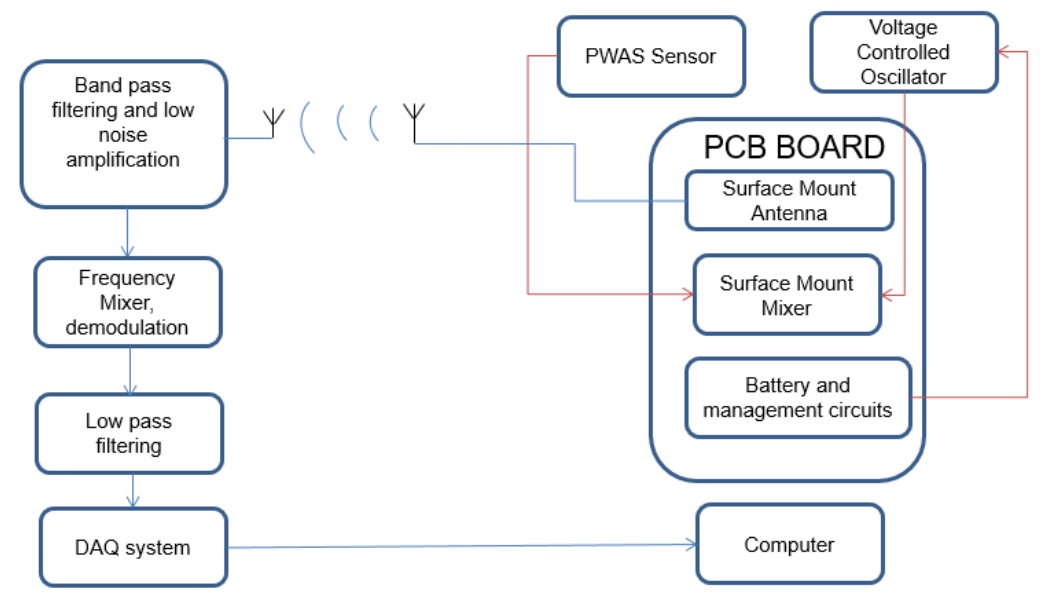

Fig 4 Schematic diagram of modified wireless transmission system

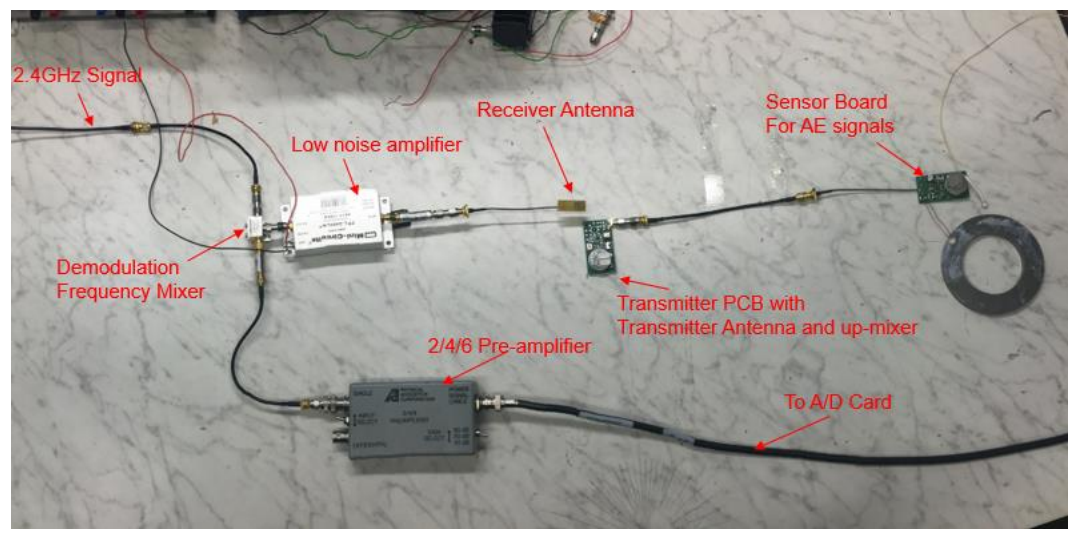

Fig 5 Modified analogue AE wireless transmission system 

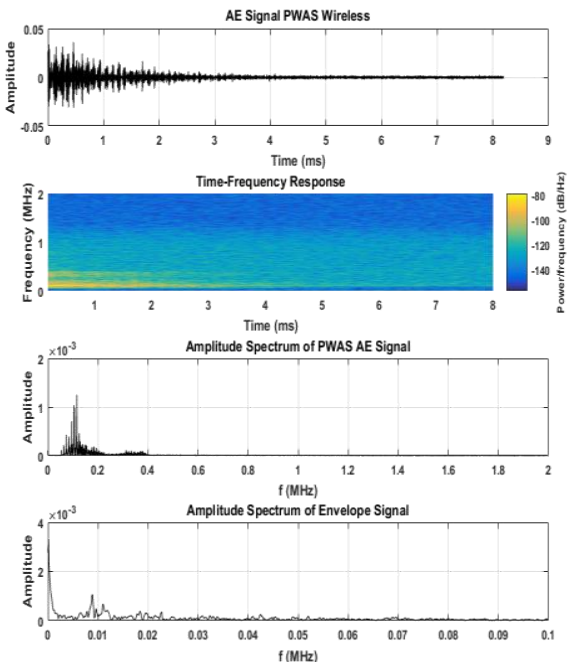
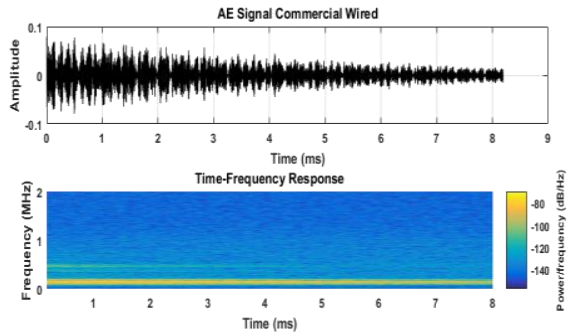

Amplitude Spectrum of Commercial Sensor AE Signal
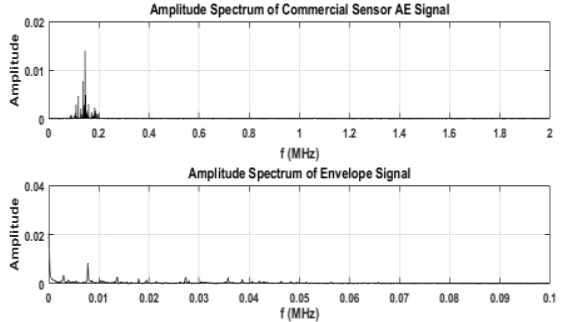

Fig 6 Pencil lead break test results of modified system

Two PCB are designed, of which one contains PWAS sensor and voltage follower for sensor buffer, amplifier selection is TLV 2781. The other board acts as transmitter that contains a voltage-controlled oscillator MAX 2750, surface mount frequency mixer SIM-73L+, and surface amount $2.4 \mathrm{GHz}$ button antenna. Both boards have embedded $3 \mathrm{~V}$ battery and power management circuits to minimise power consumption. Voltage controlled oscillator generates $2.4 \mathrm{GHz}$ carrier signal, which then is mixed with IF signal from PWAS sensor. Up-mixed signal is transmitted wirelessly by surface amount antenna, and received at the receiver end. The processes after are the same with original design of two-antenna system. Fig 5 demonstrates modified analogue AE wireless transmission system set up. Transmitter part which will be installed at rotating components now comprises of two PCB boards and one antenna pair.

Same pencil lead break test was performed at modified one antenna pair system. The results in Fig 6 from test with modified AE wireless transmission system have demonstrated better and more reliable results. compared with result in Fig 3, the signal-to-noise ratios of wireless signal in Fig 6 is visually improved. Examining processed results, wireless AE system picks up frequency contents that are similar to that of cabled commercial sensor system. Their envelopes are depicted with parallel pattern. As obviously noted, test results showed that the modified system reacts fast to excitations, and envelope of wireless AE signal has demonstrated anticipated response similar to cable-transferred signal.

\section{Conclusions}

A wireless $\mathrm{AE}$ transmission system specifically targeting application of $\mathrm{AE}$ techniques in rotating machines is proposed. Two-antenna pair system was tested with large antennas and different antenna angles to validate system effectiveness and operational angles; improvements of such system have been made, thus modified system with one pair antennas can potentially be fit inside rotating machine such as gearboxes. Bench tests have shown promising results, which indicate that such system can pick up AE signals when excited, and transmit AE signal with proper quality. Picking AE event correctly is crucial for application of fault detection when using processing method such as envelope analysis. Future work is testing modified system inside commercial helicopter main gearbox, and validating system's performance when working under harsh condition inside noisy operational gearbox. 


\section{References}

Carpinteri, A., Lacidogna, G., \& Pugno, N., 2007. Structural damage diagnosis and life-time assessment by acoustic emission monitoring. Engineering Fracture Mechanics, 74(1), 273-289.

Mba, D., \& Rao, R. B. (2006). Development of Acoustic Emission Technology for Condition Monitoring and Diagnosis of Rotating Machines; Bearings, Pumps, Gearboxes, Engines and Rotating Structures.

Al-Ghamd, A. M., \& Mba, D., 2006. A comparative experimental study on the use of acoustic emission and vibration analysis for bearing defect identification and estimation of defect size. Mechanical systems and signal processing, 20(7), 1537-1571.

Greaves, M., Elasha, F., Mba, D et. al., 2012. VHM Vibration health or alternative monitoring technologies for helicopters, EASA.2012.OP.13, U.K.

Grosse, C.U. and Krüger, M., 2006. Wireless acoustic emission sensor networks for structural health monitoring in civil engineering. In Proc. European Conf. on Non-Destructive Testing (ECNDT), DGZfP BB-103-CD.

Lédeczi, Á., Hay, T., Volgyesi, P., Hay, D.R., Nádas, A. and Jayaraman, S., 2009. Wireless acoustic emission sensor network for structural monitoring. IEEE Sensors Journal, 9(11), pp.1370-1377.

Islam, M.M., Zahedi, F. and Huang, H., 2013, April. Battery-less PWAS-based wireless Acoustic Emission sensor. In SPIE Smart Structures and Materials+ Nondestructive Evaluation and Health Monitoring (pp. 86924C-86924C). International Society for Optics and Photonics.

Huang, H. and Islam, M., 2012, April. PWAS-based wireless acoustic emission sensor. In SPIE Smart Structures and Materials+ Nondestructive Evaluation and Health Monitoring (pp. 834512-834512). International Society for Optics and Photonics. 